\title{
Thermodynamics is more powerful than the role to it reserved by Boltzmann-Gibbs statistical mechanics
}

\author{
Constantino Tsallis ${ }^{1,2, a}$ and Leonardo J.L. Cirto ${ }^{1, b}$ \\ ${ }^{1}$ Centro Brasileiro de Pesquisas Fisicas (CBPF) and National Institute of Science and Technology for Complex Systems (INCT-SC), Rua \\ Dr. Xavier Sigaud 150, 22290-180 Rio de Janeiro-RJ, Brazil \\ 2 Santa Fe Institute, 1399 Hyde Park Road, Santa Fe, NM 87501, USA
}

\begin{abstract}
We briefly review the connection between statistical mechanics and thermodynamics. We show that, in order to satisfy thermodynamics and its Legendre transformation mathematical frame, the celebrated Boltzmann-Gibbs (BG) statistical mechanics is sufficient but not necessary. Indeed, the $N \rightarrow \infty$ limit of statistical mechanics is expected to be consistent with thermodynamics. For systems whose elements are generically independent or quasi-independent in the sense of the theory of probabilities, it is well known that the BG theory (based on the additive BG entropy) does satisfy this expectation. However, in complete analogy, other thermostatistical theories (e.g., $q$-statistics), based on nonadditive entropic functionals, also satisfy the very same expectation. We illustrate this standpoint with systems whose elements are strongly correlated in a specific manner, such that they escape the BG realm.
\end{abstract}

\section{Introduction}

As an enshrined scientist, Einstein, in 1949, expressed his appreciation of classical thermodynamics, thus sharing the deep impression this theory has had upon him. In his words [1,2]:

A theory is the more impressive the greater the simplicity of its premises is, the more different kinds of things it relates, and the more extended is its area of applicability. Therefore the deep impression that classical thermodynamics made upon me. It is the only physical theory of universal content concerning which I am convinced that, within the framework of applicability of its basic concepts, it will never be overthrown.

Thermodynamics is the theory of everyday phenomena. Many of its variables (volume, pressure, temperature, viscosity) and a large part of its applications (refrigerator, steam engine, batteries) are known by both scientists and nonscientists. Although we usually speak about thermodynamical laws, thermodynamic itself is not a set of fundamental Laws of Nature in exactly the same sense that Newton's law and Maxwell's equations are. We refer to the fact that thermodynamics is consistent with all such laws and, in some sense, covers them all [3]. We may say that it is the theory which is more widely connected with all fundamental physical laws, and it finds its way into many scientific fields, from elementary particles to large scale astrophysics. The main concern of classical thermodynamic is the relationship between macroscopic variables, as, for instance, the BoyleMariotte law $P \propto 1 / V$. Today it is understood that the form of its relations is consistent with the underling microscopic laws governing the constituents of the system. It is in many ways universal, and crucial aspects of it are, remarkably enough, valid regardless the particular model. In between the level of the microscopic description of a physical system and the level of its thermodynamical macroscopic relations there is statistical mechanics.

The goal of statistical mechanics is, starting from the microscopic natural rules (classical, relativistic, quantum mechanics, chromodynamics) and adequately using probability theory, to arrive to the thermodynamical relations. Along these connections between the macro- and micro- worlds, the ultimate link is made through the fundamental concept of entropy. This finding, accomplished against a stream of criticism, surely is one of the most powerful and fruitful breakthroughs of the history of physical sciences. It was achieved by Boltzmann in the last three decades of the nineteenth century. His result, currently known by every pure and applied scientist, and carved on his tombstone in Vienna, namely,

$$
S_{B G}=k_{B} \ln W
$$

is the mathematical link between the microscopically fine description (represented by $W$, the total number of accessible microscopic states of the system) and the macroscopic measurable quantities (represented by the entropy $S_{B G}$, the very same quantity introduced by Clausius in order to complete thermodynamics!). Equation (1) has been explicitly stated in this form for the first time by Planck, but was clearly known by Boltzmann. The index $G$ stands for Gibbs, who put Boltzmann's ideas forward and overspread the (classical) statistical mechanics concepts through his seminal book [4]. Equation (1) is a particular instance of a more general one, namely

$$
S_{B G}=-k_{B} \sum_{i=1}^{W} p_{i} \ln p_{i}
$$

When every microstate is equally probable, i.e., when $p_{i}=1 / W \forall i$, we recover Eq. (1). Evidently quantum mechanics was unknown to Boltzmann and it was just birthing when Gibbs' book was published. It was left to von Neumann to extend Eq. (2)

\footnotetext{
a email: tsallis@cbpf.br

b email: cirto@cbpf.br
} 
in order to encompass quantum systems. He showed that the entropy for a quantum system should be expressed by using the density matrix operator $\widehat{\rho}$, namely

$$
S_{B G}=-k_{B} \operatorname{Tr}[\widehat{\rho} \ln \widehat{\rho}]
$$

sometimes referred to as the Boltzmann-Gibbs-von Neumann entropy. Notice indeed that the above equation recovers Eq. (2) when $\widehat{\rho}$ is diagonal.

The optimization of the entropy with appropriate constraints provides the thermal equilibrium distribution, namely the BG exponential distribution, whose consequences are consistent with classical thermodynamics. In what follows we shall, however, see that entropic functionals different from the BG one must be used in order to satisfy thermodynamics for complex systems which violate the probabilistic independence (or quasi-independence) hypothesis on which the BG entropy is generically based. This is typically the case whenever there is breakdown of ergodicity.

\section{More about entropy}

The fundamental bridge between the macroscopic thermodynamical variables and the microscopic world is the entropy. Within statistical mechanics the entropy is a functional (of the probabilities) whereas within classical thermodynamics, as originally imagined by Clausius, it is the state function demanded by the Second Law. However, after Shannon's insight within the theory of communications, entropy is no longer a concept exclusively related to classical thermodynamics. In some sense we may say that the entropic functional has its own life. Nevertheless, when dealing with the entropy as the bridge linking the microscopic and the macroscopic worlds, there are constraints that bind the functional to be used as the physically appropriate entropy. Herein we focus on the mathematical expression of the entropy by imposing the constraint that it must be an extensive quantity, i.e., proportional to the system size $N$. Why should this be so as a thermodynamical requirement will be addressed below, in Section 3.

It is straightforward to verify, using Eq. (1), the extensivity of the entropy when a physical system belongs to the so called exponential class, meaning by this those systems whose number of admissible microstates increases exponentially with $N$, like $W(N) \sim \mu^{N}(\mu>1)$ in the $N \rightarrow \infty$ limit. Those systems generically exhibit weak correlations between their elements, including, as a limiting case, the probabilistically independent systems, those with no correlations at all (e.g., a classical ideal gas, or a set of noninteracting spins). Moreover, it is algebraically very simple to verify that $S_{B G}$ is not only extensive for systems of the exponential class, but also additive, according to Penrose's definition [5]. Indeed, if $A$ and $B$ are two probabilistically independent systems (hence $p_{i j}^{A+B}=p_{i}^{A} p_{j}^{B}$ for every pair $i, j$, consequently $W^{A+B}=W^{A} W^{B}$ ), we obtain $\left(k_{B}=1\right.$ henceforth):

$$
S_{B G}(A+B)=-\sum_{i, j=1}^{W} p_{i j}^{A+B} \ln p_{i j}^{A+B}=S_{B G}(A)+S_{B G}(B)
$$

To restrict ourselves only to systems of the exponential class appears as a rather limiting and generically unjustified assumption. Indeed, strong correlations between the $N$ elements do exist in a great variety of natural, artificial and social systems. One can have, for example, systems belonging to the so called power-law class, with the number of admissible microstates increasing like $W(N) \sim N^{\rho}(\rho>0)$. For this class, the additive entropy $S_{B G}$ is clearly not extensive, since it is proportional to ln $N$. Notice that $N^{\rho} \ll \mu^{N}$ for large $N$, which is intuitive since correlations tend to bind the system to a smaller number of accessible microstates. In order to ensure extensivity for this kind of systems we shall use instead the generalization [6-8] of the BG entropy given by $(q \in \mathcal{R})$ :

$$
S_{q}=\sum_{i=1}^{W} p_{i} \ln _{q} \frac{1}{p_{i}}=-\sum_{i=1}^{W} p_{i}^{q} \ln _{q} p_{i}=-\sum_{i=1}^{W} p_{i} \ln _{2-q} p_{i}
$$

In the $q \rightarrow 1$ limit, $S_{q}$ recovers $S_{B G}$ as seen in Eq. (2); $\ln _{q} x \equiv\left(x^{1-q}-1\right) /(1-q)$ (with $\left.\ln _{1} x=\ln x\right)$ is the $q$-generalized logarithm.

A remarkable property of $S_{q}$ is that it can be made extensive for the power-law class with a suitable choice of the parameter $q$. If we look at its extremum value, also occurring when the probabilities are equal, i.e., when $p_{i}=1 / W(N), \forall i$, which leads to $S_{q}=\ln _{q} W(N)$, it is straightforward to verify that, if $W(N) \sim N^{\rho}, S_{q} \sim N$ as long as $q=1-1 / \rho$, result that can not be achieved with $S_{B G}$.

Most entropic functionals different from the Boltzmann-Gibbs one are nonadditive. But it is precisely this nonadditivity which generically enables the entropy of the system to be extensive. There is in the literature a bit of confusion at this respect ${ }^{1}$ (see, for instance, [10,11]). Equation (4) expresses the additivity of $S_{B G}$, whereas the nonadditivity of $S_{q}$ is seen, as we may readily check, in

$$
S_{q}(A+B)=S_{q}(A)+S_{q}(B)+(1-q) S_{q}(A) S_{q}(B)
$$

Inspired in the above result the so-called $q$-algebra $[12,13]$ emerged. In particular, for equal probabilities, we verify the additivelike property $S_{q}\left(W^{A} \otimes_{q} W^{B}\right)=S_{q}\left(W^{A}\right)+S_{q}\left(W^{B}\right)$, where the $q$-product $\otimes_{q}$ is defined in such way that $\ln _{q}\left(x \otimes_{q} y\right)=$ $\ln _{q} x+\ln _{q} y$.

1 The confusion arises from the fact that, occasionally, some authors inadvertently use nonadditive entropies for systems for which the entropy to be used evidently is the BG one. In the words of Tirnakli "It is like trying to play golf with a soccer ball, and then complaining that it does not fit in the holes" [9]. 
Let us now focus on the stretched-exponential class, another example of systems with strong correlations between its $N$ elements, where the number of admissible microscopic configurations increases like $W(N) \sim \nu^{N^{\gamma}}(\nu>1 ; 0<\gamma<1)$. Therefore, the phase space is more restricted than the exponential class albeit being less restricted than the power-law one, i.e., $N^{\rho} \ll \nu^{N^{\gamma}} \ll \mu^{N}$ for large $N$. The (nonadditive) entropy which is able to provide extensivity for this class is ${ }^{2}(\delta>0)$ :

$$
S_{\delta}=\sum_{i=1}^{W} p_{i}\left(\ln \frac{1}{p_{i}}\right)^{\delta}
$$

Once again, the extremum of this entropic functional occurs for equal probabilities and it is straightforward to evaluate that $S_{\delta}=[\ln W(N)]^{\delta}$ in this case. For the specific value of $\delta=1 / \gamma$ we verify that $S_{\delta} \sim N$, hence extensive, a property which is unattainable with $S_{B G}$ or $S_{q}$ for correlations within the stretched-exponential class.

We may now unify $S_{q}$ and $S_{\delta}$ to form a new two-parameter entropic functional, namely, $S_{q, \delta}=\sum p_{i}\left[\ln _{q}\left(1 / p_{i}\right)\right]^{\delta}$ with $S_{1, \delta}=S_{\delta}, S_{q, 1}=S_{q}$ and $S_{1,1}=S_{B G}$, but we will not enter into details about this point here (see [14, 15]). However it is worth to mention that $S_{q, \delta}$ can be also discussed within the framework of two-parameter entropies advanced by Hanel and Thurner in $[17,18]$; see also Tempesta [19].

The three classes of correlations discussed above, together with their respective mathematical entropic functionals which yield an extensive entropy, are summarized in Table 1.

Table 1: Entropic functionals and classes of systems (exponential, power-law and stretched-exponential, see text) for which the entropy is extensive, i.e., proportional to the number $N$ of elements. $W(N)$ is the number of admissible microscopic configurations of a system with $N$ elements (only configurations with nonvanishing occurrence probability are considered admissible). We also see the specific values of $q$ and $\delta$ for which respectively $S_{q}$ and $S_{\delta}$ are extensive.

\begin{tabular}{|c|c|c|c|}
\hline & \multicolumn{3}{|c|}{ ENTROPY } \\
\hline $\begin{array}{c}W(N) \\
(N \rightarrow \infty)\end{array}$ & $\begin{array}{c}S_{B G} \\
\text { (ADDITIVE) }\end{array}$ & $\begin{array}{c}S_{q} \\
(q \neq 1) \\
\text { (NONADDITIVE) }\end{array}$ & $\begin{array}{c}S_{\delta} \\
(\delta \neq 1) \\
\text { (NONADDITIVE) }\end{array}$ \\
\hline $\begin{array}{c}\sim \mu^{N} \\
(\mu>1)\end{array}$ & EXTENSIVE & NONEXTENSIVE & NONEXTENSIVE \\
\hline $\begin{array}{c}\sim N^{\rho} \\
(\rho>0)\end{array}$ & NONEXTENSIVE & $\begin{array}{l}\text { EXTENSIVE } \\
(q=1-1 / \rho)\end{array}$ & NONEXTENSIVE \\
\hline $\begin{array}{c}\sim \nu^{N^{\gamma}} \\
(\nu>1 \\
0<\gamma<1)\end{array}$ & NONEXTENSIVE & NONEXTENSIVE & $\begin{array}{l}\text { EXTENSIVE } \\
\qquad(\delta=1 / \gamma)\end{array}$ \\
\hline
\end{tabular}

\section{Why should the thermodynamical entropy always be extensive?}

In this Section we will briefly summarize, along lines similar to those of [14, 20,21], the thermodynamic foundations underling the notion that the entropy must always be an extensive quantity. Let us first write a general Legendre-transformation form of a thermodynamical energy $G$ of a generic $d$-dimensional system ( $d$ being an integer or fractal dimension):

$$
\begin{aligned}
G(V, T, p, \mu, H, \ldots) & =U(V, T, p, \mu, H, \ldots)-T S(V, T, p, \mu, H, \ldots)+ \\
+ & p V-\mu N(V, T, p, \mu, H, \ldots)-H M(V, T, p, \mu, H, \ldots)-\cdots
\end{aligned}
$$

where $T, p, \mu, H$ are the temperature, pressure, chemical potential, external magnetic field, and $U, S, V, N, M$ are the internal energy, entropy, volume, number of particles, magnetization. We may identify three types of variables, namely (i) those that are expected to always be extensive $(S, V, N, M, \ldots)$, i.e., scaling with $V \propto L^{d}$, where $L$ is a characteristic linear dimension of the system (notice the presence of $N$ itself within this class), (ii) those that characterize the external conditions under which

\footnotetext{
2 This entropy was first proposed in [8] (footnote on page 69) in order to construct an extensive entropy for the stretched-exponential class, and has been discussed in detail in [14]. The same functional form was also discovered (independently) by Ubriaco in [16].
} 
the system is placed $(T, p, \mu, H, \ldots)$, scaling with $L^{\theta}$, and (iii) those that represent energies $(G, U)$, scaling with $L^{\epsilon}$. Ordinary thermodynamical systems are those with $\theta=0$ and $\epsilon=d$, therefore both the energies and the generically extensive variables scale with $L^{d}$ and there is no difference between the types (i) and (iii) variables, being all of them extensive in this case. There are, however, physical systems where $\epsilon=\theta+d$ with $\theta \neq 0$. Let us divide Eq. (7) by $L^{\theta+d}$, namely:

$$
\frac{G}{L^{\theta+d}}=\frac{U}{L^{\theta+d}}-\frac{T}{L^{\theta}} \frac{S}{L^{d}}+\frac{p}{L^{\theta}} \frac{V}{L^{d}}-\frac{\mu}{L^{\theta}} \frac{N}{L^{d}}-\frac{H}{L^{\theta}} \frac{M}{L^{d}}-\cdots
$$

If we consider now the thermodynamical $L \rightarrow \infty$ limit, we obtain

$$
\widetilde{g}=\widetilde{u}-\widetilde{T} s+\widetilde{p} v-\widetilde{\mu} n-\widetilde{H} m-\cdots
$$

where, using a compact notation, $(\widetilde{g}, \widetilde{u}) \equiv \lim _{L \rightarrow \infty}(G, U) / L^{\theta+d}$ represent the energies; $(s, v, n, m) \equiv \lim _{L \rightarrow \infty}(S, V, N, M) / L^{d}$ represent the usual extensive variables and $(\widetilde{T}, \widetilde{p}, \widetilde{\mu}, \widetilde{H}) \equiv \lim _{L \rightarrow \infty}(T, p, \mu, H) / L^{\theta}$ correspond to the usually intensive ones. For a standard thermodynamical system (e.g., a real gas ruled by a Lennard-Jones short-ranged potential, a simple metal, etc) we have $\theta=0$ (hence $(\widetilde{T}, \widetilde{p}, \widetilde{\mu}, \widetilde{H})=(T, p, \mu, H)$, i.e., the usual intensive variables), and $\epsilon=d$ (hence $(\widetilde{g}, \widetilde{u})=(g, u), i . e$, the usual extensive variables); this is of course the case found in the textbooks of thermodynamics. Not yet really explored in textbooks are those cases with $\theta \neq 0$. Indeed, the correctness of the scaling appearing in Eq. (9) for nonstandard systems, i.e., for those with $\theta \neq 0$, has been profusely verified for several systems in the literature [22-36]; one of them is going to be discussed in Section 4 below. Furthermore, it has been shown that such scalings preserve important thermodynamical relations such as the Euler and Gibbs-Duhem [21].

The thermodynamic relations (7) and (8) put on an equal footing the entropy $S$, the volume $V$ and the number of elements $N$, and there can be no doubt about the extensivity of the latter two variables. In fact, similar analysis can be performed using $N$ instead of $V$ since $V \propto N$.

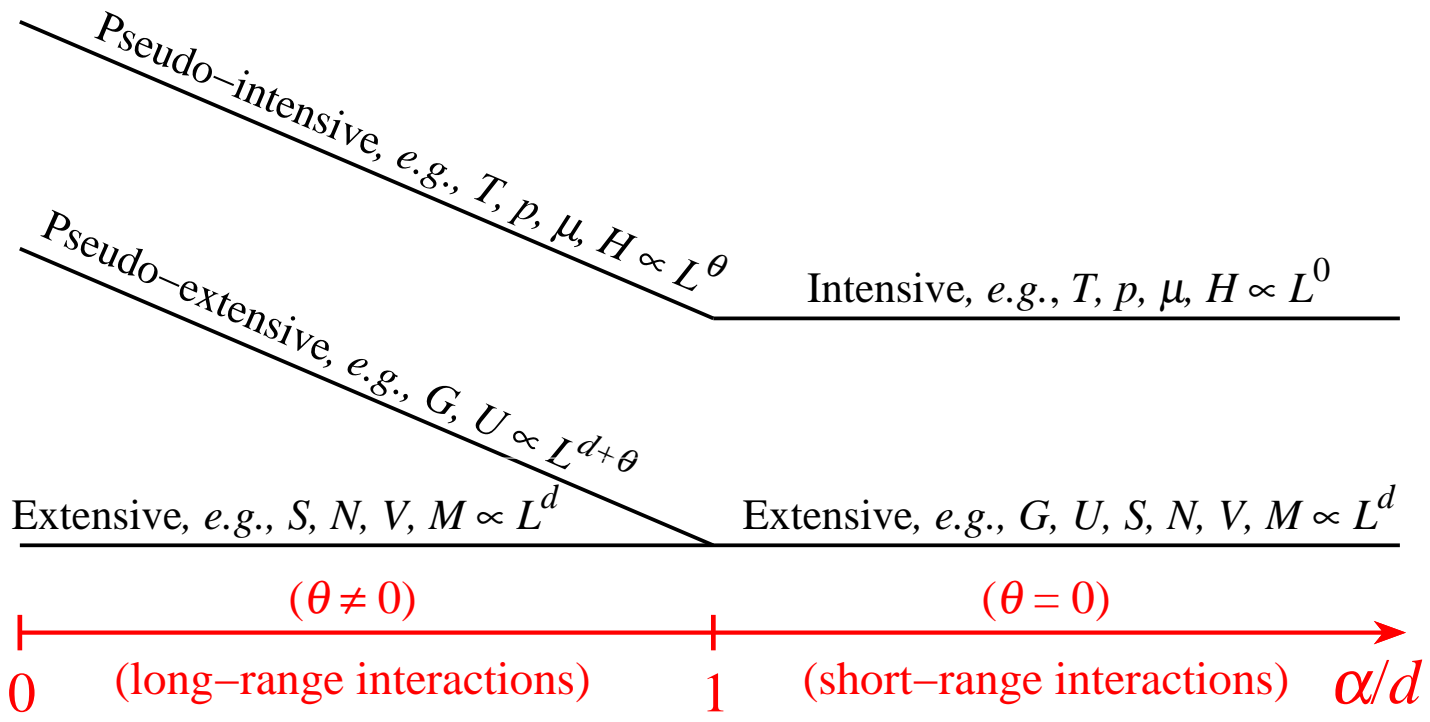

Figure 1: Representation of the different scaling regimes of the Eq. (8) for classical $d$-dimensional systems. For attractive long-range interactions (i.e., $0 \leq \alpha / d \leq 1, \alpha$ characterizes the interaction range in a potential with the form $1 / r^{\alpha}$ ) we may distinguish three classes of thermodynamic variables, namely, those scaling with $L^{\theta}$, named pseudo-intensive ( $L$ is a characteristic linear length, $\theta$ is a system-dependent parameter), those scaling with $L^{d+\theta}$, the pseudo-extensive ones (the energies), and those scaling with $L^{d}$ (which are always extensive). For short-range interactions (i.e., $\alpha>d$ ) we have $\theta=0$ and the energies recover their standard $L^{d}$ extensive scaling, falling in the same class of $S, N, V$, etc, whereas the previous pseudo-intensive variables become truly intensive ones (independent of $L$ ); this is the region, with two classes of variables, that is covered by the traditional textbooks of thermodynamics.

An example of nonstandard system with $\theta \neq 0$ is the classical Hamiltonian discussed in Section 4 below. We consider twobody interactions decaying with distance $r$ like $1 / r^{\alpha}(\alpha \geq 0)$. For this system we have $\theta=d-\alpha$ whenever $0 \leq \alpha<d$ (see, for example, Fig. 1 of [26]). This peculiar scaling occurs because the potential is not integrable, i.e., the integral $\int_{\text {constant }}^{\infty} d r r^{d-1} r^{-\alpha}$ diverges for $0 \leq \alpha \leq d$, therefore the Boltzmann-Gibbs canonical partition function itself diverges. Gibbs was aware of this kind of problem and has pointed out [4] that whenever the partition function diverges, the BG theory can not be used because, in his words, "the law of distribution becomes illusory". The divergence of the total potential energy occurs for $\alpha \leq d$, which is referred to as long-range interactions. If $\alpha>d$, which is the case of the $d=3$ Lennard-Jones potential, whose attractive part corresponds to $\alpha=6$, the integral does not diverge and we recover the standard behaviour of short-range-interacting systems with the $\theta=0$ scaling. Nevertheless, it is worth recalling that nonstandard thermodynamical behaviour is not necessarily associated with longrange interactions in the classical sense just discussed. A meaningful description would then be long-range correlations (spatial 
or temporal) because for strongly quantum-entangled systems, correlations are not necessarily connected with the interaction range (see Section 5). However the picture of long- versus short-ranged interactions in the classical sense, directly related to the distance $r$, has the advantage to depict clearly the thermodynamic relations (7) and (8) for the different scaling regimes, as shown in Fig. 1.

One more recent result is now available [37-39], related to the so called Large Deviation Theory in theory of probabilities [40, 41], which also is consistent with the extensivity of the entropy, even in the presence of strong correlations between the elements of the system. In fact it is known since several decades that the mathematical foundation of BG statistical mechanics crucially lies on the theory of large deviations. To attain the same status for nonextensive statistical mechanics, it is necessary to $q$-generalize the large deviation theory itself. The purpose of those efforts precisely is to make a first step towards that goal through the study of a simple model.

Finally, a further indication we can refer to is the analogy with the time $t$ dependence of the entropy of simple nonlinear dynamical systems, e.g., the logistic map. Indeed, for the parameter values for which the system has positive Lyapunov exponent (i.e., strong chaos and ergodicity), we verify $S_{B G} \propto t$ (under appropriate mathematical limits), but for parameter values where the Lyapunov exponent vanishes nontrivially, e.g., the Feigenbaum point (i.e., weak chaos), it is the nonadditive entropy $S_{q}$ for a specific value of $q$ the one which grows linearly with $t$ (see [42-51] and references therein), and consistently provides a generalized Pesin-like identity. If we take into account that, in many such dynamical systems, $t$ plays a role analogous to $N$ in thermodynamical systems, we have here one more indication which aligns with the extensivity of the entropy for complex systems.

In what follows we illustrate the above concepts through three physical systems, namely a long-range-interacting many-body classical Hamiltonian system (Section 4), a strongly quantum entangled system at zero temperature (Section 5), and black holes (Section 6).

\section{A classical model with long- and short- ranged interactions}

To better discuss the concepts of long- and short-range interaction let us see a concrete and well known example, namely, an ensemble of $N$ classical spins arranged in a lattice whose Hamiltonian is given by

$$
\mathcal{H}=-J \sum_{\langle i, j\rangle}^{N} \mathbf{S}_{i} \cdot \mathbf{S}_{j}
$$

where $J>0$ is the ferromagnetic coupling constant. The symbol $\langle i, j\rangle$ means that the sum runs only over the nearest neighbour for each spin. If the system lies in a ring, i.e., a one-dimensional system, each spin has only two nearest neighbours; if it lies in say bidimensional plane and is arranged as a square lattice there are four nearest neighbours. It is a typical example of what is referred to as short-range interactions. Depending on the dimension of the spin vector $\mathbf{S}_{i}$, the Hamiltonian (10) may represent the Ising, the classical XY or Heisenberg models, all of them very well understood and described by the traditional Boltzmann-Gibbs equilibrium statistical mechanics (see, for example, the classical paper by Stanley [52] for the linear case).

Let us now consider the case where the interaction is not restricted to the nearest neighbours anymore. Let us consider the other extreme situation where all spins interact with all others with the same strength regardless the distance between them. The system is said to be fully-coupled and is described by the following Hamiltonian:

$$
\mathcal{H}=-J \sum_{i=1}^{N} \sum_{j=1}^{N} \mathbf{S}_{i} \cdot \mathbf{S}_{j}
$$

It is a typical example of a long-range system. It may be directly assessed that the total energy of this model is not proportional to the system size $N$, hence the system is nonextensive and, strictly speaking, there is no traditional thermostatistics in this case at all. In the framework of Section 3, Hamiltonian (11) is associated with $\theta=d$ which means a $U \propto V^{2 d} \propto N^{2}$ scaling. Accordingly, there is no quantity different from zero or from infinity that can be calculated ("the law of distribution becomes illusory" here).

A way to overcome the difficulty without moving out of the standard formalism can be thought of. If the (initially) constant coupling constant is conveniently rescaled as $J \rightarrow J / N$ - nowadays called Kac's prescription —, the extensivity of the system is recovered. Mathematically this procedure is evidently rightful but it throws us in an strange situation where the microscopic coupling constant $J$ become dependent on $N$, i.e., following Baxter's words [53], it leave us with the "unphysical property that the interaction strength depends on the number of particles".

Leaving aside the epistemological issue of having a two-body coupling constant $J$ dependent on the system $N$ let us put forward the scaling $J \rightarrow J / N$ idea and explore the following system [25, 26, 32, 54, 55]:

$$
\mathcal{H}=\frac{1}{2} \sum_{i=1}^{N} p_{i}^{2}+\frac{J}{2 \widetilde{N}} \sum_{i=1}^{N} \sum_{\substack{j=1 \\ j \neq i}}^{N} \frac{1-\cos \left(\theta_{i}-\theta_{j}\right)}{r_{i j}^{\alpha}}
$$

The Hamiltonian (12) is a extension of the models (10) and (11) by including a kinetic term provided that the classical spin vectors $\mathbf{S}_{i}$ be bidimensional, since, in this case, $\mathbf{S}_{i} \cdot \mathbf{S}_{j}=\cos \left(\theta_{i}-\theta_{j}\right)$. In the $\alpha \rightarrow \infty$ limit, the interaction term of Eq. (12) 
approaches the first-neighbours Hamiltonian (10), whereas if $\alpha=0$ it approaches the fully-coupled instance (11). The latter case is called Hamiltonian Mean Field, or simply HMF, after Antoni and Ruffo' s work [54], a model that has been profusely studied in the past years.

The parameter $\widetilde{N}$ is judiciously chosen in order to make the energy extensive for all values of $\alpha / d$. It behaves, when $N$ is large, like $\tilde{N} \sim N^{1-\alpha / d}$ if $0 \leq \alpha / d<1$ and like $\tilde{N} \sim \mathcal{O}(1)$ if $\alpha / d>1$. In other words, it recovers the general scaling for systems with $\alpha \neq 0$. This is referred to as the $\alpha-\mathrm{XY}$ model (the spins may be visualized as XY-planar rotators), and it is a genuine Hamiltonian system in the sense that the variables $p_{i}$ and $\theta_{i}$ are canonical conjugate pairs. With a kinetic term the model presents its own dynamics and equation of motion can be derived throughout a Hamiltonian formulation. Consequently, to enquire numerically physical properties of the system through molecular dynamic simulations constitutes a natural route.

After scaling the interaction with $\widetilde{N}$, the energy of the system becomes extensive and all the traditional thermodynamical techniques can be applied (the canonical partition function does not diverge in the thermodynamic limit anymore). It remains, however, nonadditive, i.e., if we bring together two system $A$ and $B$ ruled by Hamiltonian (12), the joined internal energy $u_{A+B}$, with $u=\langle\mathcal{H} / N\rangle$, will be $u_{A+B} \neq u_{A}+u_{B}$ in general. This happens because the long-range nature of the interaction is still present, property particularly seen when $\alpha=0$ and the scaling decreases the interaction strength equally regardless the distance. Thereby, even with the extensivity recovered, unexpected behaviour should not be seen as striking, and it has been actually caught in several (numerical) experiments. As one example of unpredict behaviour within the traditional scenario, one has the long-lived quasistationary states (QSS) which emerges for $\alpha / d<1$ [32,56-66]. In these states the thermodynamical quantities like temperature and magnetization do not coincide with the canonical predictions. Moreover, its lifetime diverge with increasing system size $N$, associated with the order in which the thermodynamic $N \rightarrow \infty$ and the infinite time $t \rightarrow \infty$ limits are considered. Specifically, if we let $N \rightarrow \infty$ first, the system remains trapped in these QSS's, never reaching the final Boltzmann-Gibbs equilibrium state, most probably being the QSS itself the ultimate state in this case. Another example comes from the one-momentum distribution. Within the BG framework it is expected a Maxwellian distribution for the velocities, no matter whether it is calculated by using time or ensemble averages. This distribution shape, i.e., a Gaussian, is in fact observed in the (time average) numerical simulations, but only for $\alpha$ sufficiently large (hence short-range). However, if $\alpha$ is small (hence long-range), it was observed [32, 65, 67] distributions very well described by $q$-Gaussians, in disagreement with the traditional BG thermostatistical scenario (see Fig. 2).

The model (12) is very rich and certainly will continue giving rise to several new and interesting results. These nonstandard behaviours observed for $\alpha / d<1$ appear to be in line with nonergodicity [66,68-70] and with the thesis of the $q$-generalized Central Limit Theorem (see references and comments in [32]). The fact that the a ad hoc scaling of the interaction recovers the formal extensivity but not eliminate its intrinsic long-range nature appears to be the reason why this model is not satisfactorily described within the BG thermodynamics scenario. It is conceivable that such long-range interaction could generate correlations strong enough to constraint the dynamics of the system within some regions of the phase space, thus reducing the "number" of accessible microstates in the same spirit of the correlation classes discussed in Section 2.

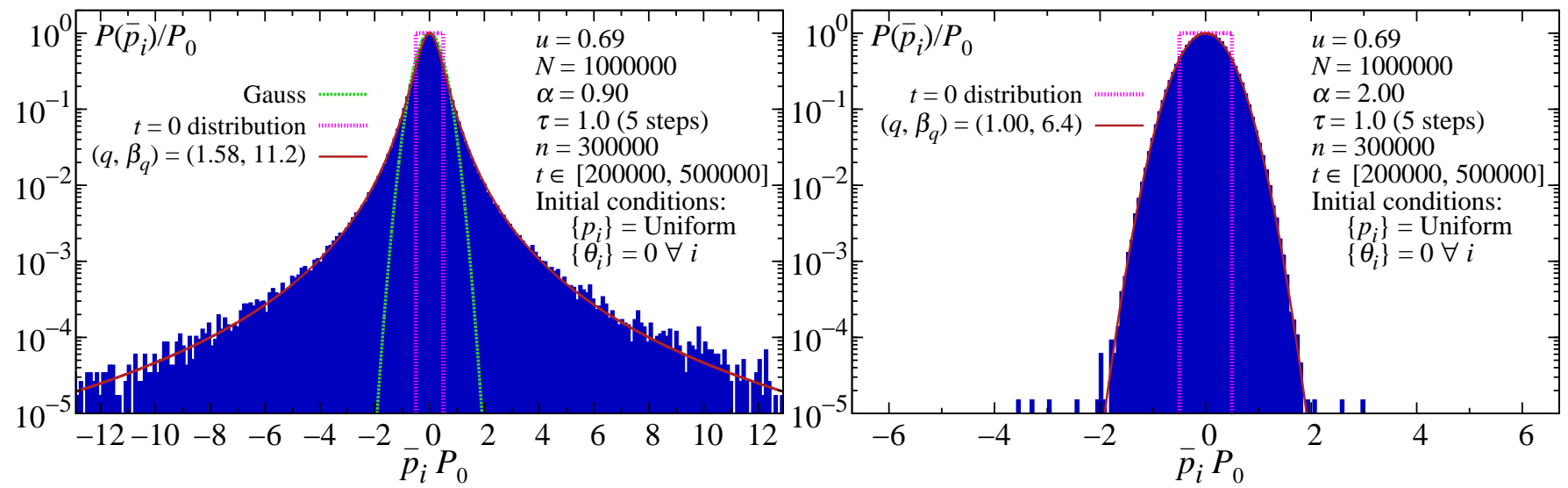

Figure 2: Molecular dynamics results for the $d=1$ Hamiltonian (12) for two values of $\alpha$ under identical simulational setup for all the other parameters (energy, initial conditions, number of particles, period over which the time average is calculated, etc; we consider here, without loss of generality, $J=1$ ). What we see is a typical single-initial-condition one-momentum distribution for $\alpha=0.9<d=1$ (long-range, left plot) and $\alpha=2.0>d=1$ (short-range, right plot). The continuous curves correspond to $q$-Gaussians with $q=1.58$ for $\alpha=0.9$ and $q=1$ (i.e., a Gaussian) for $\alpha=2.0$. See details in [32].

\subsection{Searching for $q$ from first principles}

It is expected that the index $q$ of the entropy functional $S_{q}$ shown in Eq. (5) to be an intrinsic property of the geometri$\mathrm{cal} /$ dynamical nature of the occupancy of phase space. It should be calculated from first principles, i.e., from the microscopic 
fundamental dynamical law governing the system. However this calculation is by no means an easy task and, in many cases, it will be virtually impossible without strong mathematical approximations. Nevertheless a few examples have been analytically solved wherein a first-principle $q$ value was achieved, as the one discussed in Section 5 bellow. Here, for the many-body $\alpha$-XY model, $q$ shall be approached through the Hamiltonian (12) itself.

Inspired by the $q$-Gaussian one-momentum distribution seen in Fig. 2 we may figure out a possible route to calculate from first principles the value of $q$. This distribution extremize the nonadditive entropy $S_{q}$ upon which the nonextensive statistical mechanics [6-8] is based. Within this framework, the stationary state is expected to yield a probability distribution $\exp _{q}\left(-\beta_{q} \mathcal{H}\right) / Z_{q}\left(\beta_{q}\right)$ with $Z_{q}\left(\beta_{q}\right)$ being the generalized partition function $\left(\exp _{q}\right.$ represents the inverse of the $q$-generalized logarithm defined in Section 2 ; the $\exp _{q}$ function becomes the ordinary exponential for $q=1$ ). The one-momentum marginal probability would then be calculated using $P\left(p_{1}\right)=\int \mathrm{d} p_{2} \ldots \mathrm{d} p_{N} \mathrm{~d} \theta_{1} \ldots \mathrm{d} \theta_{N} \exp _{q}\left(-\beta_{q} \mathcal{H}\right) / Z_{q}$. The possible functional form of $P\left(p_{1}\right)$ could be a $q_{m}{ }^{-}$ Gaussian, where $m$ stands for momentum (we singled the label out here because the value $q_{m}$ is not necessarily the same as that of the entropic functional $S_{q}$; naturally we expect $q_{m}=1$ if $q=1$ ). The entropic index $q$ (and also $q_{m}$ ) is expected to characterize universality classes, possibly a function $q=q(\alpha / d)$ to be different from 1 for $0 \leq \alpha / d<1$, and equal to 1 for $\alpha / d \geq 1$ in accordance with the numerical experiments. At the present computational stage, we have access to $q_{m}$ but not yet to $q$. The latter implies an extremely heavy computational task since it has to do with the occupancy of the entire many-body phase space for given initial conditions.

\section{A fully quantum-entangled system - An exact calculation of $q$ from first principles}

When dealing with classical systems, spatial correlations and long-time memory are usually neatly connected with long-range interactions. However, when one goes deeper in the microscopic structure of the matter the strictly quantum mechanical phenomenon of entanglement comes into play and long-range correlations are not necessarily connected with long-range interactions in the sense discussed in the Section 4. For example, let us consider the following first-neighbourhood interaction Hamiltonian describing a quantum $N$ spin-1/2 ferromagnetic chain under a transverse magnetic field at its critical value at zero temperature:

$$
H=-\sum_{i=1}^{N-1}\left[(1+\gamma) \sigma_{i}^{x} \sigma_{i+1}^{x}+(1-\gamma) \sigma_{j}^{y} \sigma_{i+1}^{y}+2 \lambda \sigma_{i}^{z}\right]
$$

where $\sigma^{\mu}, \mu=x, y, z$, are the Pauli's matrices, and $\gamma$ and $\lambda$ are the intensity of the anisotropy and magnetic field respectively. Known as quantum XY model, Hamiltonian (13) recovers for $|\gamma|=1$ (i.e., maximum axial anisotropy) the quantum Ising chain. Furthermore it is known that, in the thermodynamic $N \rightarrow \infty$ limit, a quantum phase transition (hence at $T=0$ ) exists at the critical point $\left|\lambda_{c}\right|=1$.

If we have complete information about a system its entropy is zero. Quantum mechanically, complete information means that we are dealing with a pure state. Evidently the intrinsic probabilistic nature of a quantum system forbids us to have complete information in a classical sense; a pure state means that there is an unique quantum state describing the system. At zero temperature the system is in its fundamental state, hence the entropy should be zero for any admissible entropic functional. Being $\widehat{\rho}_{N}$ the density operator of the whole chain, a pure state means that $\operatorname{Tr} \widehat{\rho}_{N}^{2}=\operatorname{Tr} \widehat{\rho}_{N}=1$. However, even for $T=0$, it is possible to calculate a entropy different from zero if we consider only a block of $L$ contiguous spins and work with the reduced matrix $\widehat{\rho}_{L}=\operatorname{Tr}_{N-L} \widehat{\rho}_{N}$. This reduced matrix in general does not represent a pure state, but a mixed state instead (i.e., $\operatorname{Tr} \widehat{\rho}_{L}^{2}<\operatorname{Tr} \widehat{\rho}_{L}=1$ ). This fact is a consequence of the nonlocal entanglement that is responsible for the long-range quantum correlations of the spin chain (13) at $T=0$.

The degree of entanglement between a block of $L$ contiguous spins and the rest of the chain in its ground state can be characterized by the von Neumann entropy (3) of the block (see [71,72]). For a large block size, it typically saturates off criticality, whereas it is logarithmically unbounded at the critical point, i.e., the so called area law for $d=1$ systems. In order words, the BG entropy at $T=0$ for the chain (13) does not scale with the system size, but like $S_{B G}(L) \propto \ln L$, being $S_{B G}(L) \propto L^{d-1}$ the general area-law scaling for $d$-dimensional systems with $d>1$. However, it was shown [73] (see also [74]) that the thermodynamical extensivity is recovered if we move from the BG entropic functional to the $S_{q}$ one. Furthermore, as the Hamiltonian (13) can be exactly diagonalized, is was possibly to calculate analytically, for the universality class characterized by the central charge $c$, a closed form for $q$, namely

$$
q=\frac{\sqrt{9+c^{2}}-3}{c}
$$

Therefore, in order to achieve an extensive entropy for the chain (13), which implies a finite value for $S / L$ in the $L \rightarrow \infty$ limit, it is enough to calculate $S_{q}$ with the specific value of $q$ shown in Eq. (14) (see Fig. 3).

\section{On the entropy for black holes}

Far from going into details on this fascinating topic, which very recently had its foundations expanded by one of its most important contributors [75,76], we will use the black hole physical system as a possible application of the entropy $S_{\delta}$ discussed in Section 2 (Eq. (6)). As already discussed, the Boltzmann-Gibbs entropy has, as underling hypothesis, weak correlations and 


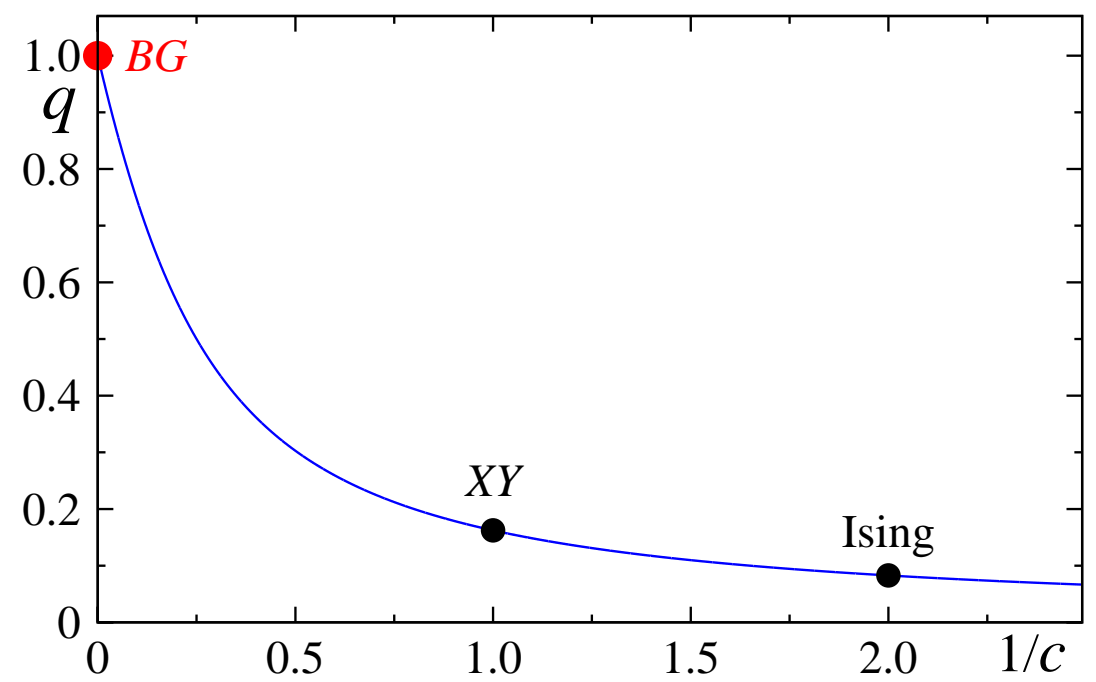

Figure 3: $q$ as a function of the central charge $c$ which characterizes the universality class (and which contains the quantum spin chain (13) as a particular instance). The BG entropy for a block of $L$ contiguous spins is $S_{B G}(L) \propto \ln L$ for all finite values of the central charge, thus violating thermodynamical extensivity. However, for the special values of $q$ shown here (see Eq. (14)), $S_{q}(L) \propto L$, i.e., it satisfies one-dimensional extensivity, thus enabling the use of all the relations that can be found in any good textbook of thermodynamics. See details in [73].

ergodicity. To fit a black hole under this general assumptions may eventually not be a safe thermodynamical starting approach. Indeed, the outstanding results of Bekenstein and Hawking [77-79] have already shown that the BG entropy of a black hole is proportional to its boundary surface. It is important to recall that, for a variety of reasons, this result appears to be evidently true, i.e., the $\mathrm{BG}$ entropy is in fact proportional do the area, as several and diversified calculations along almost forty years have confirmed [80]. In a few words, the Bekenstein-Hawking result reads:

$$
S_{B H} \propto A
$$

where $A$ is the event horizon area. If the black hole is to be considered as a genuine $d=2$ system, which means that it is physically identified solely with its event horizon surface, Eq. (15) is extensive and must be seen as the the truly thermodynamical entropy. Therefore, in a thermodynamical sense, there is nothing that should be regarded as intriguing or unusual, and, strictly speaking, this would not be an area-law problem [72,81-83]. However, if the black hole is to be considered a genuine $d=3$ system, we then recover the very same discussion of the previous Section 5. Its thermodynamical entropy then should not be associated with the additive BG functional and a nonadditive generalization should be used instead. It happens that the Bekenstein-Hawking result (15) is very helpful here too, since it says to us that $W \propto e^{b A}(b>0)$, hence we are dealing with a stretched-exponential class system. Therefore, it follows that extensivity is recovered by using the entropic functional $S_{\delta}$ with $\delta=3 / 2$ (see more details in [32]).

This idea has recently been put forward by Komatsu and Kimura [84, 85] within a entropic-force scenario. See also [86], where the probability distribution that extremizes $S_{\delta}$ and its associated Fokker-Plank equation are analyzed.

\section{Final remarks}

To conclude, let us now summarize the line of thought that we have presented here. Thermodynamics is a highly valuable approach to nature, and we see no reason at all for generalizing its basic principles, in particular in what concerns entropy. Because of the Legendre-transform structure of thermodynamics, as well as because of strong indications within the probabilistic large-deviation theory, the extensivity of the entropy must be preserved in all cases that we are aware of, whether short- or longranged-interacting systems, classical or quantum (strongly entangled or not), dissipative systems, among others. For systems that live in their entire (or nearly entire) phase space (or Hilbert or Fock spaces if the system is a quantum one), in other words, if the system is essentially ergodic in a region with finite Lebesgue measure, the number $W(N)$ of admissible microscopic possibilities increases exponentially with $N$ (exponential class). Consequently it is the Boltzmann-Gibbs (additive) entropic functional which guarantees the extensivity of the entropy. But if, due to strong correlations between the elements of the system, the occupancy of phase space is severely restricted (so strongly that the Lebesgue measure of the visited region is zero, which is the case of the power-law and stretched-exponential classes), we typically need nonadditive entropic functionals such as $S_{q}$ or $S_{\delta}$ in order to comply with the requirement of extensivity for the thermodynamical entropy. This fact has very relevant consequences, in particular in what concerns the probability distributions that spontaneously emerge in the corresponding (frequently unique) stationary or quasi-stationary states. They tend to exhibit, for example for the power-law class, $q$-exponential behaviors (which asymptotically are power-laws) instead of the usual exponential ones that typically are observed for all kinds of relevant physical 
properties. A vast literature illustrates this scenario [87]. We have here selected a few of such examples (classical long-rangeinteracting many-body Hamiltonian systems, strongly quantum entangled systems at their quantum critical point, black holes). The same picture is seen in many other systems through analytical, experimental, observational and computational results in natural, artificial and social systems, along with predictions, verifications and applications (see, for instance, a brief review in [88]). Further reinforcing and clarifying evidences are naturally welcome. Indeed, a variety of open questions (whose details are out from the present scope) still remain to be better understood.

\section{Acknowledgments}

We have benefited from fruitful remarks by M. Jauregui and U. Tirnakli. We also acknowledge partial financial support from CNPq, Faperj and Capes (Brazilian agencies). It is our great pleasure to dedicate this review to Hans J. Herrmann, wishing him a very happy anniversary for his (first) 60 years!

\section{References}

1. A. Einstein, Notes for an autobiography. The Saturday Review, November 26, 9-12 and 36-44 (1949)

2. M.J. Klein, Thermodynamics in Einstein's Thought. Science 157, 509-516 (1967)

3. A.S. Eddington, The Nature of the Physical World (The Macmillan Company, New York 1929) page 74. In his words: "The law that entropy always increases, holds, I think, the supreme position among the laws of Nature. If someone points out to you that your pet theory of the universe is in disagreement with Maxwell's equations-then so much the worse for Maxwell's equations. If it is found to be contradicted by observation-well, these experimentalists do bungle things sometimes. But if your theory is found to be against the second law of thermodynamics, I can give you no hope; there is nothing for it but to collapse in deepest humiliation".

4. J.W. Gibbs, Elementary Principles in Statistical Mechanics - Developed with Especial Reference to the Rational Foundation of Thermodynamics (C. Scribner's Sons, New York 1902); Yale University Press, New Haven 1948; OX Bow Press, Woodbridge, Connecticut 1981)

5. O. Penrose, Foundations of statistical mechanics: A deductive treatment (Pergamon, Oxford 1970) page 167

6. C. Tsallis, Possible generalization of Boltzmann-Gibbs statistics. J. Stat. Phys. 52, 479-487 (1988)

7. M. Gell-Mann, C. Tsallis, Editors, Nonextensive entropy - Interdisciplinary applications (Oxford University Press, New York 2004). In the title there is a misname: it should be "Nonadditive Entropy".

8. C. Tsallis, Introduction to nonextensive statistical mechanics - Approaching a complex world (Springer, New York 2009)

9. U. Tirnakli, private communication (2014)

10. S. Pressé, K. Ghosh, J. Lee, K.A. Dill, Nonadditive entropies yield probability distributions with biases not warranted by the data. Phys. Rev. Lett. 111, 180604 (2013)

11. C. Tsallis, Comment on "Nonadditive entropies yield probability distributions with biases not warranted by the data" by Pressé et al. arXiv:1404.1257v1 [cond-mat.stat-mech] (2014)

12. E.P. Borges, A possible deformed algebra and calculus inspired in nonextensive thermostatistics. Physica A 340, 95-101 (2004)

13. L. Nivanen, A. Le Méhauté, Q.A. Wang, Generalized algebra within a nonextensive statistics. Rep. Math. Phys. 52, $437-444$ (2003)

14. C. Tsallis, L.J.L. Cirto, Black hole thermodynamical entropy. Eur. Phys. J. C 73, 2487 (2013)

15. M.S. Ribeiro, F.D. Nobre, C. Tsallis, Probability distributions and associated nonlinear Fokker-Planck equation for the two-index entropic form $S_{q, \delta}$. Phys. Rev. E 89, 052135 (2014)

16. M.R. Ubriaco, Entropies based on fractional calculus. Phys. Lett. A 373, 2516-2519 (2009)

17. R. Hanel, S. Thurner, A comprehensive classification of complex statistical systems and an axiomatic derivation of their entropy and distribution functions. Europhys. Lett. 93, 20006 (2011)

18. R. Hanel, S. Thurner, When do generalized entropies apply? how phase space volume determines entropy. Europhys. Lett. 96, 50003 (2011)

19. P. Tempesta, Group entropies, correlation laws, and zeta functions. Phys. Rev. E 84, 021121 (2011)

20. C. Tsallis, Nonextensive thermodynamics and fractals. Fractals 03, 541-547 (1995)

21. S. Abe, A.K. Rajagopal, Scaling relations in equilibrium nonextensive thermostatistics. Phys. Lett. A 337, 292-295 (2005)

22. P. Jund, S.G. Kim, C. Tsallis, Crossover from extensive to nonextensive behavior driven by long-range interactions. Phys. Rev. B 52, 50-53 (1995)

23. S.A. Cannas, F.A. Tamarit, Long-range interactions and nonextensivity in ferromagnetic spin models. Phys. Rev. B 54, R12661-R12664 (1996)

24. L.C. Sampaio, M.P. de Albuquerque, F.S. de Menezes, Nonextensivity and tsallis statistics in magnetic systems. Phys. Rev. B 55, 5611-5614 (1997)

25. C. Anteneodo, C. Tsallis, Breakdown of exponential sensitivity to initial conditions: Role of the range of interactions. Phys. Rev. Lett. 80, 5313-5316 (1998)

26. F. Tamarit, C. Anteneodo, Rotators with long-range interactions: Connection with the mean-field approximation. Phys. Rev. Lett. 84, 208-211 (2000)

27. A. Giansanti, D. Moroni, A. Campa, Universal behaviour in the static and dynamic properties of the $\alpha-X Y$ model. Chaos, Solitons \& Fractals 13, 407-416 (2002)

28. Ch. Binek, S. Polisetty, Xi He, T. Mukherjee, R. Rajesh, J. Redepenning, Nonextensivity in magnetic nanoparticle ensembles. Phys. Rev. B 74, 054432 (2006)

29. J.R. Grigera, Extensive and non-extensive thermodynamics. A molecular dynamic test. Phys. Lett. A 217, 47-51 (1996) 
30. S. Curilef, C. Tsallis, Critical temperature and nonextensivity in long-range-interacting Lennard-Jones-like fluids. Phys. Lett. A 264, 270-275 (1999)

31. R.F.S. Andrade, S.T.R. Pinho, Tsallis scaling and the long-range ising chain: A transfer matrix approach. Phys. Rev. E 71, 026126 (2005)

32. L.J.L. Cirto, V.R.V. Assis, C. Tsallis, Influence of the interaction range on the thermostatistics of a classical many-body system. Physica A 393, 286-296 (2014)

33. L.J.L. Cirto, L.S. Lima, F.D. Nobre, Controlling the range of interactions in the classical inertial ferromagnetic Heisenberg model: Analysis of metastable states. arXiv:1409.5825 [cond-mat.stat-mech] (2014)

34. C.A. Condat, J. Rangel, P.W. Lamberti, Anomalous diffusion in the nonasymptotic regime. Phys. Rev. E 65, 026138 (2002)

35. H.H.A. Rego, L.S. Lucena, L.R. da Silva, C. Tsallis, Crossover from extensive to nonextensive behavior driven by long-range $d=1$ bond percolation. Physica A 266, 42-48 (1999)

36. U.L. Fulco, L.R. da Silva, F.D. Nobre, H.H.A. Rego, L.S. Lucena, Effects of site dilution on the one-dimensional long-range bondpercolation problem. Phys. Lett. A 312, 331-335 (2003)

37. G. Ruiz, C. Tsallis, Towards a large deviation theory for strongly correlated systems. Phys. Lett. A 376, 2451-2454 (2012)

38. H. Touchette, Comment on "Towards a large deviation theory for strongly correlated systems". Phys. Lett. A 377, 436-438 (2013)

39. G. Ruiz, C. Tsallis, Reply to comment on "Towards a large deviation theory for strongly correlated systems". Phys. Lett. A 377, 491-495 (2013)

40. R.S. Ellis, Entropy, Large Deviations, and Statistical Mechanics (Springer, New York 1985).

41. H. Touchette, The large deviation approach to statistical mechanics. Phys. Rep. 478, 1-69 (2009)

42. U. Tirnakli, C. Tsallis, M.L. Lyra, Circular-like maps: Sensitivity to the initial conditions, multifractality and nonextensivity. Eur. Phys. J. B 11, 309-3015 (1999)

43. G. Ruiz, C. Tsallis, Nonextensivity at the edge of chaos of a new universality class of one-dimensional unimodal dissipative maps. Eur. Phys. J. B 67, 577-584 (2009)

44. F. Baldovin, A. Robledo, Sensitivity to initial conditions at bifurcations in one-dimensional nonlinear maps: Rigorous nonextensive solutions. Europhys. Lett. 60, 518-524 (2002)

45. G. Casati, C. Tsallis, F. Baldovin, Linear instability and statistical laws of physics. Europhys. Lett. 72, 355-361 (2005)

46. E.P. Borges, C. Tsallis, G.F.J. Ananos, P.M.C. de Oliveira, Nonequilibrium probabilistic dynamics at the logistic map edge of chaos. Phys. Rev. Lett. 89, 254103 (2002)

47. G.F.J. Ananos, C. Tsallis, Ensemble averages and nonextensivity at the edge of chaos of one-dimensional maps. Phys. Rev. Lett. 93, 020601 (2004)

48. F. Baldovin, A. Robledo, Universal renormalization-group dynamics at the onset of chaos in logistic maps and nonextensive statistical mechanics. Phys. Rev. E 66, R045104 (2002)

49. F. Baldovin, A. Robledo, Nonextensive Pesin identity: Exact renormalization group analytical results for the dynamics at the edge of chaos of the logistic map. Phys. Rev. E 69, 045202(R) (2004)

50. E. Mayoral, A. Robledo, Tsallis' $q$ index and Mori's q phase transitions at edge of chaos. Phys. Rev. E 72, 026209 (2005)

51. E. Mayoral, A. Robledo, Multifractality and nonextensivity at the edge of chaos of unimodal maps. Physica A 340, 219-226 (2004)

52. H.E. Stanley, Exact solution for a linear chain of isotropically interacting classical spins of arbitrary dimensionality. Phys. Rev. 179, 570-577 (1969)

53. R.J. Baxter, Exactly Solved Models in Statistical Mechanics (Academic Press Inc., London 1982) page 39. Freely available with author's permission at http://physics.anu.edu.au/theophys/baxter_book.php.

54. M. Antoni S. Ruffo, Clustering and relaxation in Hamiltonian long-range dynamics. Phys. Rev. E 52, 2361-2374 (1995)

55. A. Campa, A. Giansanti, D. Moroni, Canonical solution of a system of long-range interacting rotators on a lattice. Phys. Rev. E 62, 303-306 (2000)

56. V. Latora, A. Rapisarda, S. Ruffo, Lyapunov instability and finite size effects in a system with long-range forces. Phys. Rev. Lett. 80, 692-695 (1998)

57. V. Latora, A. Rapisarda, S. Ruffo, Chaos and statistical mechanics in the Hamiltonian mean field model. Physica D 131, 38-54 (1999)

58. V. Latora, A. Rapisarda, C. Tsallis, Non-gaussian equilibrium in a long-range Hamiltonian system. Phys. Rev. E 64, 056134 (2001)

59. A. Campa, A. Giansanti, D. Moroni, Metastable states in a class of long-range Hamiltonian systems. Physica A 305, 137-143 (2002)

60. A. Pluchino, V. Latora, A. Rapisarda, Glassy dynamics in the HMF model. Physica A 340, 187-195 (2004)

61. A. Pluchino, V. Latora, A. Rapisarda, Metastable states, anomalous distributions and correlations in the HMF model. Physica D 193, 315-328 (2004)

62. A. Pluchino, V. Latora, A. Rapisarda, Effective spin-glass Hamiltonian for the anomalous dynamics of the HMF model. Physica A 370, 573-584 (2006)

63. Y.Y. Yamaguchi, J. Barré, F. Bouchet, T. Dauxois, S. Ruffo, Stability criteria of the Vlasov equation and quasi-stationary states of the HMF model. Physica A 337, 36-66 (2004)

64. L.G. Moyano, C. Anteneodo, Diffusive anomalies in a long-range Hamiltonian system. Phys. Rev. E 74, 021118 (2006)

65. A. Pluchino, A. Rapisarda, C. Tsallis, A closer look at the indications of q-generalized central limit theorem behavior in quasi-stationary states of the HMF model. Physica A 387, 3121-3128 (2008)

66. W. Ettoumi, M.-C. Firpo, Action diffusion and lifetimes of quasistationary states in the Hamiltonian mean-field model. Phys. Rev. E 87, 030102 (2013)

67. A. Pluchino, A. Rapisarda, C. Tsallis, Nonergodicity and central-limit behavior for long-range Hamiltonians. Europhys. Lett. 80, 26002 (2007)

68. A. Campa, T. Dauxois, S. Ruffo, Statistical mechanics and dynamics of solvable models with long-range interactions. Phys. Rep. 480, 57-159 (2009)

69. P.-H. Chavanis, A. Campa, Inhomogeneous Tsallis distributions in the HMF model. Eur. Phys. J. B 76, 581-611 (2010)

70. A. Campa, P.-H. Chavanis, Caloric curves fitted by polytropic distributions in the HMF model. Eur. Phys. J. B 86, 170 (2013) 
71. L. Amico, R. Fazio, A. Osterloh, V. Vedral, Entanglement in many-body systems. Rev. Mod. Phys. 80, $517-576$ (2008)

72. J. Eisert, M. Cramer, M.B. Plenio, Colloquium: Area laws for the entanglement entropy. Rev. Mod. Phys. 82, 277-306 (2010)

73. F. Caruso, C. Tsallis, Nonadditive entropy reconciles the area law in quantum systems with classical thermodynamics. Phys. Rev. E 78, $021102(2008)$

74. A. Saguia, M.S. Sarandy Nonadditive entropy for random quantum spin-S chains. Phys. Lett. A 374, 3384-3388 (2010)

75. S.W. Hawking, Information preservation and weather forecasting for black holes. arXiv:1401.5761v1 [hep-th] (2014)

76. Z. Merali, Stephen Hawking: "There are no black holes". Nature News 24 January (2014)

77. J.D. Bekenstein, Black holes and entropy. Phys. Rev. D 7, 2333 (1973)

78. S.W. Hawking, Black hole and thermodynamics. Phys. Rev. D 13, 191 (1976)

79. J.D. Bekenstein, Black holes and information theory. Contemp. Phys. 45, 31-43 (2004)

80. S.N. Solodukhin, Entanglement entropy of black holes. Living Rev. Relativity 14, 8 (2011)

81. S. Das, S. Shankaranarayanan, How robust is the entanglement entropy-area relation? Phys. Rev. D 73, 121701 (2006)

82. R. Brustein, M.B. Einhorn, A. Yarom, Entanglement interpretation of black hole entropy in string theory. J. High Energy Phys. 2006, 098 (2006)

83. S. Kolekar, T. Padmanabhan, Ideal gas in a strong gravitational field: Area dependence of entropy. Phys. Rev. D 83, 064034 (2011)

84. N. Komatsu, S. Kimura, Entropic cosmology for a generalized black-hole entropy. Phys. Rev. D 88, 083534 (2013)

85. N. Komatsu, S. Kimura, Evolution of the universe in entropic cosmologies via different formulations. Phys. Rev. D 89, 123501 (2014)

86. M.S. Ribeiro, C. Tsallis, F.D. Nobre, Probability distributions extremizing the nonadditive entropy $S_{\delta}$ and stationary states of the corresponding nonlinear Fokker-Planck equation. Phys. Rev. E 88, 052107 (2013)

87. A regularly updated bibliography can be seen at http://tsallis.cat.cbpf.br/biblio.htm.

88. C. Tsallis, An introduction to nonadditive entropies and a thermostatistical approach to inanimate and living matter. Contemp. Phys. 55, 179-197 (2014) 